primary care with the purpose to expand and strengthen DOTS in $\mathrm{TB}$ high burden municipalities aiming the decentralisation of TB care.

Method Two TB burdened metropolitan regions were chosen for TB Training: (a) Metropolitan Area of Sao Paulo (approximately 20 million inhabitants) and (b) Sao Paulo Atlantic Coast (approximately 2 million inhabitants) with 18 high TB burden municipalities. Sao Paulo State has a huge population distributed in its 645 municipalities with 73 high TB burden cities. The training model used a framework consisting of classes and workgroups for 5 workdays $(20 \mathrm{~h})$, with a participative methodology to propitiate an interaction between trainees and tutors generating discussions and allowing the consensus in TB-related matters.

Results The training activities took place in 2010 sponsored by Sao Paulo State Secretary of Health and Global Fund TB Brazil. The TB training has been accomplished successfully with $1256 \mathrm{HCW}$ from May to October 2010. The 2009 epidemiological evaluation achieved active case findings rates from $0.9 \%$ to $126.6 \%$ of goal which is to examine $1 \%$ of inhabitants of each municipality, and treatment coverage rates on DOT from $7.8 \%$ to $96.8 \%$, demonstrating the need of improving rates in some TB high burden municipalities regarding TB trainings of HCWs.

Conclusions Training activities must be understood as a planning and follow-up resource in order to improve TB control activities.

\section{SP6-68 MALARIA IN PREGNANT WOMEN IN HIGH AND MEDIUM INCIDENCE AREAS IN NIAS DISTRICT, INDONESIA, 2005}

doi:10.1136/jech.2011.142976q.39

I Winarta.* Indonesia University, Depok, West Java, Indonesia

Background Malaria is a major problem which is serious in pregnancy. Pregnant women are more often exposed to malaria infection compared to non-pregnant women. The prevalence of malaria in pregnant women around the world is $10 \%-65 \%$. In pregnancy malaria on the pregnant women are that it can cause anaemia, death, infant miscarriage, infant death, and low birth weight. The aim of this research was to describe the proportion of pregnant women with malaria and describe the influence of factors on high incidence areas and medium incidence areas in Nias district, Indonesia.

Methods This research used a cross-sectional design with primer data. A sample was taken from pregnant women who had not taken anti-malaria medication with the last month. 440 pregnant women were sampled, 220 pregnant women in a medium incidence area and 220 in high incidence area. The sample was taken by multistage random sampling. Analyses were conducted to describe the proportion and determinant factors in each area. Research variables were malaria, gravity, parity, stage of pregnancy age, mother's age, occupation, knowledge about malaria, usage of insect killers and closed outpits

Results The proportion of pregnant women with malaria in the High Incidence Area (HIA) was $36.36 \%$ and Medium Incidence Area (MIA) $31.36 \%$ and HIA+MIA $33.86 \%$. The proportion of pregnant women that had suffered a clinical symptom in the last month in HIA was $10.90 \%$ and in MIA $35.45 \%$.

Conclusion Factors determining malaria prevalence in pregnant women in HIA and MIA are gravity, knowledge, usage of insect killer and closed outpits. The importance of treatment and how to protect from malaria can be achieved by having a standard book on malaria, blood examination during antenatal care and health promotion.

\section{SP6-69 A STUDY OF REPORTED SICKNESS PATTERN OF AIR PASSENGERS AT INDIAN AIRPORTS}

doi:10.1136/jech.2011.142976q.40

B K Singh.* Airports Authority of India, New Delhi, South East Asia, India

There are 58 million air passengers using 125 airports in India in 2009-2010. Out of which $58 \%$ are in domestic sector $42 \%$ in international sector. There is no centralised data of morbidity of air travellers. Air passengers request for medical assistance are given free help at all airports managed by AAI. Other 7 joint venture airports charge for medical assistance.

Method Records of Air passenger asking for help their disposal has been analysed for year 2009

Result Pattern is one reported sick passenger per 23000 in international travel and 1 in 44000 in domestic sector. Most of passengers $(50 \%)$ of reported sickness were seen in departure in domestic while arrival cases $(55 \%)$ were more in international sector. $10 \%$ cases were sent to hospitals for opinion and treatment. There were 67 non-schedule landing due to medical reasons at all airports. There were three emergency landing due to disruptive air passenger in Indian Sky. There were 12 deaths at airports. On analysing symptoms Diarrhoea, Giddiness, Chest pain, Choking, Fits, earache, toothache, bleeding from natural orifices, minor injuries, accidents, others. Gender wise males were more. Age wise middle and geriatric age group asked for medical help.

Cause Probably Nomadic Stress Complex and tolerance limit played some role.

Conclusion Air travel morbidity pattern is not fully known till date. A need for Tele Medicine for international air passengers is felt. A proper protocol for handling disruptive air passenger at airports/in sky is felt especially for international citizen.

\section{SP6-70 NATIONAL HEALTH INSURANCE SCHEME, MDG AND MATERNAL AND CHILD HEALTH IN OYO STATE, NIGERIA}

doi:10.1136/jech.2011.142976q.41

${ }^{1} 0$ Omobowale, ${ }^{2} 0$ Omobowale. ${ }^{1}$ University College Hospital, Ibadan, Oyo, Nigeria; ${ }^{2}$ University of Ibadan, Ibadan, Oyo, Nigeria

Lack of adequate healthcare financing is one of the factors that prevent women from getting the healthcare they need. To remove the financial barrier to maternal and child health services for women in the non-formal sector, the National Health Insurance SchemeMillenium Development Goal and Maternal and Child Health Project established. The study was to determine the factors affecting the coverage and utilisation of the NHIS-MDG MCH project in Oyo State, Nigeria. A cross sectional facility-based empirical study was carried out. Both secondary and primary data were collected through the assessment of non-confidential records such as NHIS and hospital records and oral key informant interviews with coordinators of NHIS-MDG/MCH project in three selected local government areas. Presently, only 100000 potential beneficiaries are expected to be covered in Oyo State, which has a population of women in the child bearing age as 1269514 and that of children under 5 years as 1154104 . Lack of adequate skilled personnel at the health facilities, poor infrastructure, delay in counterpart funding by the state government, political influence, poverty, as well as poor community awareness are factors affecting the coverage and utilisation of the NHIS-MDG/MCH project. Level of coverage and utilisation of the NHIS-MDG/MCH services was low. Strategies to improve awareness, coverage and utilisation of these services among women and children in the non-formal sector should be implemented. 